Effect of sequence dispersity on morphology of tapered diblock copolymers from molecular dynamics simulations

William G. Levine, Youngmi Seo, Jonathan R. Brown, and Lisa M. Hall

Citation: J. Chem. Phys. 145, 234907 (2016); doi: 10.1063/1.4972141

View online: http://dx.doi.org/10.1063/1.4972141

View Table of Contents: http://aip.scitation.org/toc/jcp/145/23

Published by the American Institute of Physics 


\title{
Effect of sequence dispersity on morphology of tapered diblock copolymers from molecular dynamics simulations
}

\author{
William G. Levine, ${ }^{1,2}$ Youngmi Seo, ${ }^{1}$ Jonathan R. Brown, ${ }^{1}$ and Lisa M. Hall ${ }^{1, a)}$ \\ ${ }^{1}$ William G. Lowrie Department of Chemical and Biomolecular Engineering, The Ohio State University, \\ 151 W. Woodruff Ave., Columbus, Ohio 43210, USA \\ ${ }^{2}$ Division of Physics, Mathematics and Astronomy 105-24, Caltech, Pasadena, California 91125, USA
}

(Received 18 September 2016; accepted 1 December 2016; published online 20 December 2016)

\begin{abstract}
Tapered diblock copolymers are similar to typical AB diblock copolymers but have an added transition region between the two blocks which changes gradually in composition from pure A to pure B. This tapered region can be varied from $0 \%$ (true diblock) to $100 \%$ (gradient copolymer) of the polymer length, and this allows some control over the microphase separated domain spacing and other material properties. We perform molecular dynamics simulations of linearly tapered block copolymers with tapers of various lengths, initialized from fluids density functional theory predictions. To investigate the effect of sequence dispersity, we compare systems composed of identical polymers, whose taper has a fixed sequence that most closely approximates a linear gradient, with sequentially disperse polymers, whose sequences are created statistically to yield the appropriate ensemble average linear gradient. Especially at high segregation strength, we find clear differences in polymer conformations and microstructures between these systems. Importantly, the statistical polymers are able to find more favorable conformations given their sequence, for instance, a statistical polymer with a larger fraction of $\mathrm{A}$ than the median will tend towards the A lamellae. The conformations of the statistically different polymers can thus be less stretched, and these systems have higher overall density. Consequently, the lamellae formed by statistical polymers have smaller domain spacing with sharper interfaces. Published by AIP Publishing. [http://dx.doi.org/10.1063/1.4972141]
\end{abstract}

\section{INTRODUCTION}

Tapered AB block copolymers have blocks of pure A and $\mathrm{B}$ monomers on each end of the polymer, separated by a transition block or "taper" whose composition changes smoothly from purely A to purely B monomers (or from B to A monomers for inverse tapers) along its length. A major driver of interest in such systems is that, by adjusting the length of the tapered region, one can tune microphase behavior and physical properties such as glass transition temperature $\left(\mathrm{T}_{\mathrm{g}}\right)$ and orderdisorder transition temperature $\left(\mathrm{T}_{\mathrm{ODT}}\right)$, as has been found in experiments by multiple groups. ${ }^{1-17}$ As would be intuitively expected, adding a taper generally increases the miscibility of the system, effectively decreasing the segregation strength (quantified by $\chi \mathrm{N}$, the Flory $\chi$ parameter times polymer length) and widens the interfacial regions of the microphase separated structures. Furthermore, tapering has been shown to change the microphase behavior and dynamics versus typical diblocks in ways that cannot be explained by a simple shift in effective segregation strength. ${ }^{16-20}$ The promise of tapered polymers was highlighted in a recent work showing that intermediate length tapers can enhance polymer dynamics, improving ion transport in lithium ion battery electrolyte materials. ${ }^{21}$

Simulation and theory can provide insight into the physical origins of such behavior and potentially provide guidance in implementing this new control parameter

a) E-mail: hall.1004@osu.edu effectively. ${ }^{17-20,22}$ Interestingly, self-consistent field theory (SCFT) predicted that short to medium length normal tapering significantly increases the region of the phase diagram where the bicontinuous double gyroid microphase is favored. ${ }^{18}$ Coarse-grained molecular dynamics (MD) simulations started from a random initial configuration generally microphase separated into the same phases predicted by SCFT. ${ }^{19}$ A combination of fluids density functional theory (fDFT) $)^{23-25}$ and MD simulations were used to further explore the effects of tapering, including the effects on polymer diffusion. ${ }^{19,20}$ Recently, Sethuraman and Ganesan also used MD simulations to study tapered copolymers; they showed how dynamic properties depend on both effective $\chi$ and the difference in A and B segmental mobility. ${ }^{22}$ In both the aforementioned SCFT and fDFT works, each polymer in a given system had an identical composition that approximates a linear gradient (a particular fixed sequence of $\mathrm{A}$ and $\mathrm{B}$ beads for the fDFT or alternating A and B blocks of varying lengths for SCFT). Meanwhile, the MD simulations used weighted random sequences such that individual polymers are different, but the overall ensemble average shows a linear gradient in the taper. Of course, some degree of sequence dispersity also exists in experimentally synthesized polymers. ${ }^{26}$ One way tapered copolymers can be formed experimentally is via a semi-batch process of many sequential living anionic polymerization steps; during each step the two different types of monomers are injected in different amounts and are polymerized approximately randomly., $4,16,27-32$ A very recent work showed an excellent agreement between tapered copolymer density profiles from experiment and 
fDFT (with a fixed sequence), ${ }^{17}$ though comparisons between experiment and theory with respect to order-disorder transitions or dynamics have not been as specific and precise.

Though the strategy of using tapering to tune material properties hinges on a detailed understanding and control over the effects of polymer sequence on overall behavior, none of these prior studies have specifically addressed the discrepancy in sequence dispersity between the theoretical, simulation, and experimental tapered systems. Meanwhile, for the related gradient copolymer systems that have been studied more frequently in the literature, sequence dispersity is known to affect both the order-disorder transition and microphase domain spacing, among other properties. ${ }^{33-36}$ In the current study, we are motivated to quantify the effects of sequence dispersity for tapered systems. We aim to show both: to what extent the theoretical work employing fixed sequences is representative of statistical sequence systems and, more generally, how sequence dispersity affects the microphase structure.

The literature showing the effect of sequence dispersity for gradient copolymers builds on the significant body of work that has been accumulated for many decades regarding the effects of molecular weight polydispersity. For a few examples of theory and simulation work in this area, molecular weight polydispersity for diblocks is explored in Ref. 37 and the effect of sequential polydispersity of graft random copolymers is shown in Refs. 38 and 39. Additionally, Refs. 40 and 41 discuss how blockiness or dispersity in block sequence impacts the phase behavior of random block copolymer melts.

Of more specific interest is prior work on the effects of sequence dispersity for gradient copolymers; when the composition gradient is linear, these systems correspond to the limit of 100\% tapering. ${ }^{33-35}$ Ref. 34 employed Monte Carlo (MC) simulations to study gradient copolymers in solution. The polymer sequences could be either "quenched" (sequentially polydisperse), in which each monomer could be A or B with a probability specified by a linear composition profile, or "annealed" (sequentially monodisperse), in which each monomer is partially A and B with a fraction determined by the same linear composition (the pairwise interaction strengths were adjusted according to the fractional monomer type). The sequentially monodisperse system required a higher interaction strength to form micelle-like structures. More recently, Shi and co-workers studied linear gradient copolymer melts, with different amounts of compositional polydispersity, using SCFT. ${ }^{33}$ They also find the interaction strength required for microphase separation is higher for the monodisperse system. Interestingly, while the compositionally monodisperse systems have smaller lamellar spacing near the critical point, with increasing $\chi \mathrm{N}$ their spacing increases more than that of polydisperse systems. Thus, strongly segregated monodisperse systems have a larger domain spacing than polydisperse systems.

Ganesan and coworkers also recently studied a range of copolymers with linear or hyperbolic composition gradients, some of which are similar to the tapered composition profiles considered here. They considered the effects of both the local sequence blockiness (average length of continuous A or B monomer regions) and compositional polydispersity (variance in the local fraction of A across all chains in the system, considering a given point along the chain length). ${ }^{35}$ Using self-consistent Brownian dynamics (SCBD) simulations, they showed that both kinds of sequential polydispersity increase the size of the microphase separated region of the phase diagram versus that expected for monodisperse systems. The effect of sequence dispersity was more important for systems with a more gradual change in composition across the polymer length.

In this study we consider linearly tapered copolymers and focus on one aspect of compositional dispersity: whether polymers in the system all have the same sequence that approximates a linear taper or all sequences are statistically determined with a linearly weighted probability in the tapered region. In the section titled Methods, we describe our detailed simulation methodology including how we defined the fixed and statistical sequence systems. In the results and discussion, we discuss how different the interfacial sharpness of lamellae and other structural, conformational properties are in the two systems with various taper lengths. These differences will be discussed by comparing to the existing simulation or theoretical results. Finally we conclude with summarizing remarks in the conclusions.

\section{METHODS}

We use a simple Kremer-Grest bead-spring model of linear polymer chains with 40 monomer beads per chain that can be of type A or B. ${ }^{42}$ Systems are symmetric with an equal amount of A and B beads overall, and the only microphase separated structure considered here is lamellae. Thus, diblock copolymers (0\% tapers) consist of $20 \mathrm{~A}$ beads followed by 20 B beads. Tapered chains are composed of an A block, a tapered region whose composition changes approximately linearly along its length from pure A to pure B (or from B to A for inverse tapers), then a B block. Specifically, 30\%, 50\%, and $70 \%$ normal tapers and $30 \%$ inverse tapers are considered. The statistical or sequentially polydisperse systems are created as in our previous MD studies. ${ }^{19,20}$ Specifically, each monomer site in the tapered region has a linearly weighted random chance of being either type A or B such that the ensemble average composition shows an ideal linear gradient in the tapered region. Each monomer site is allocated with $\left[(\mathrm{i}-0.5) / \mathrm{N}_{\mathrm{t}}\right] \times 960$ number of A monomers, where $\mathrm{i}$ is the site number along the taper, $\mathrm{N}_{\mathrm{t}}$ is the number of beads in the tapered block, and 960 is the total number of polymers, and which beads appear on which polymer chains is determined through random shuffling by site. In such systems, individual polymer chains have distinct sequences of beads and the fraction of A beads on a given chain $\left(f_{A}\right)$ is not necessarily 0.5 . For compositionally monodisperse systems, a single, fixed sequence of $\mathrm{A}$ and $\mathrm{B}$ is used for all chains in the simulation; see Figure 1 for the particular sequences considered in this paper. As shown in Figure 2, the chosen sequences best match a perfectly linear taper in terms of the cumulative number of A beads as a function of distance along the taper, as in our prior fDFT work. ${ }^{17,20}$

Monomers are bonded using the finitely extensible nonlinear elastic (FENE) potential, Equation (1), with a spring constant of $k=30 \varepsilon / \sigma^{2}$ and maximum length of $R_{0}=1.5 \sigma$ to 
(a) (A) $14-A-A-A-B-A-B-A-B-A-B-B-B-(B)_{14}$

(b) (A) 10 -A-A-A-A-B-A-A-B-A-B-A-B-A-B-B-A-B-B-B-B-(B) 10 (c) $(A)_{6}-A-A-A-A-A-B-A-A-B-A-A-B-A-A-B-B-A-B-B-A-B-B-A-B-B-B-B-B-(B)_{6}$ (d) $(A)_{14}-B-B-B-A-B-A-B-A-B-A-A-A-(B)_{14}$

FIG. 1. Monomer sequences employed for fixed sequence systems: (a) $30 \%$ taper, (b) 50\% taper, (c) $70 \%$ taper, and (d) $30 \%$ inverse taper. All individually written As and Bs are considered part of the taper; parentheses are used to indicate the pure monomer blocks.

appropriately avoid chain crossing or breaking, ${ }^{42}$

$$
U_{F E N E}(r)=-0.5 k R_{0}^{2} \ln \left(1-\frac{r^{2}}{R_{0}^{2}}\right) .
$$

The purely repulsive part of the Lennard-Jones (LJ) potential (the standard LJ potential cutoff and shifted to 0 at $r_{c}=2^{1 / 6} \sigma$ ) is used for all pairwise interactions,

$$
U_{L J}(r)=\left\{\begin{array}{cc}
4 \varepsilon_{i j}\left[\left(\frac{\sigma_{i j}}{r}\right)^{12}-\left(\frac{\sigma_{i j}}{r}\right)^{6}+\frac{1}{4}\right] & r \leq r_{c} \\
0 & r>r_{c}
\end{array},\right.
$$

where $\varepsilon_{i j}$ is the interaction strength and $\sigma_{i j}$ is the length scale of interaction between monomers $i$ and $j$. All $\sigma_{i j}=1.0 \sigma$, and $\varepsilon_{i j}$ is equal for like monomer interactions $\left(\varepsilon_{A A}=\varepsilon_{B B}=1.0 \varepsilon\right) ; \sigma$ and $\varepsilon$ are used as our units for length and energy, respectively.

We considered $\varepsilon_{A B}$ values of 3.0, 4.0, 5.0, 6.1, 7.4, 9.0, and 11.9 for normal tapers and 6.1, 7.0, 7.4, 8.0, 9.0, 10.0, $10.6,11.0,11.5$, and 11.9 for inverse tapers, depending on the system. Some of these values were used in our prior work, and the chosen values are more closely spaced for inverse tapers to show more clearly their lamellar spacing as a function of $\varepsilon_{A B}$, which was shown to change subtly and sometimes non-monotonically in our prior work. ${ }^{18}$ At the highest value considered (11.9), all systems are clearly microphase separated to lamellae. Some systems tend to disorder at the lower values

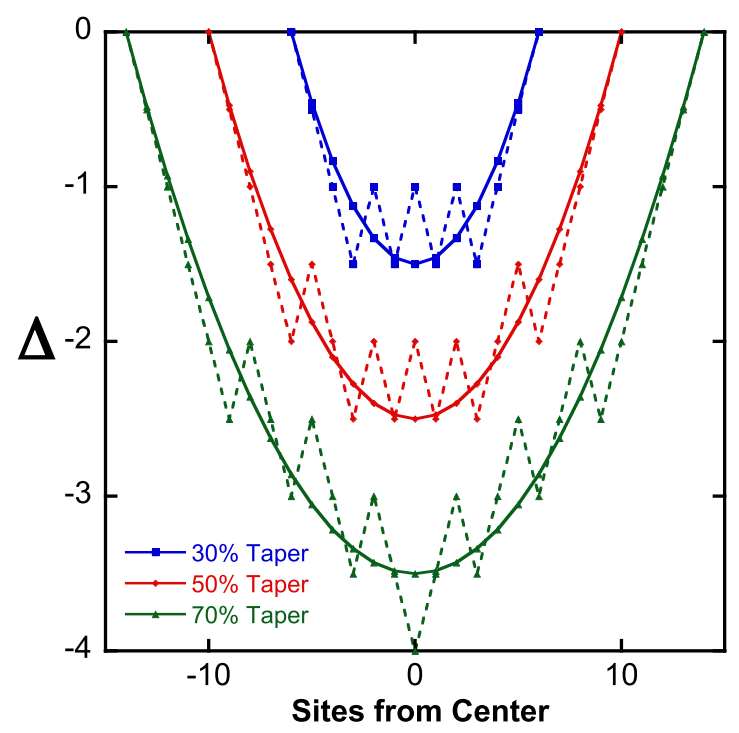

FIG. 2. Expected cumulative count of B beads found along the tapered region, relative to the expected value along a fully random sequence with equal amounts of $\mathrm{A}$ and $\mathrm{B}, \Delta$, plotted as a function of site number along the chain and shifted such that 0 represents the count to the center of the taper. The tapered region is $30 \%, 50 \%$, or $70 \%$ of the total length of 40 beads, as labeled. Points connected by dashed lines show the depletion of A beads for the chosen fixed sequences as shown in Figure 1, while smooth lines show the expected value for an ideal linear gradient, which is a parabola centered in the middle of the taper. The fixed sequences were chosen to best match the ideal linear gradient. of $\varepsilon_{A B}$; we report results below only for systems that keep their 8-layered lamellar structure over the whole simulation time of $230000 \tau$, based on visual inspection. Specifically, lamellae did not appear stable for $\varepsilon_{A B}=4.0$ for $70 \%$ normal tapers and for $\varepsilon_{A B}=7.0$ for $30 \%$ inverse tapers with fixed sequences, and these and lower values of $\varepsilon_{A B}$ for each system are not included below. A simple linear mapping to $\chi$ as reported in Ref. 20 suggests that $\chi=0.39\left(\varepsilon_{A B}-1\right)$. This implies that $\chi N \approx 31$ and 170 for the lowest and highest considered values of $\varepsilon_{A B}=3.0$ and 11.9 , respectively. However, both Refs. 20 and 43 suggest that at large repulsive interaction strengths, the mapping is no longer linear and the effective $\chi$ is somewhat lower than expected. While one can perform a more detailed mapping, for clarity we discuss our results below simply with respect to the interaction parameter $\varepsilon_{A B}$.

All simulations are initialized in lamellar structures to avoid the need to simulate over the long time scales relevant to microphase separation. Each system contains 8 lamellae, aligned in the $\mathrm{z}$ direction and with equal numbers of polymers in each. The initial polymer configurations are created based on fDFT results for the analogous model system (with the same sequence as used in the fixed sequence MD systems) at the same $\varepsilon_{A B}$. Briefly, polymers are grown via a pseudo-random walk starting at the interface, where the likelihood of placing a monomer at a particular $\mathrm{z}$ value depends on its fDFT density profile; the details are identical to those reported in Ref. 20. All systems have 960 polymers in total, thus 120 polymers per lamellar interface.

Starting from the initial fDFT-informed lamellar configuration, the simulation proceeds with a brief push-off with a soft potential, $A\left[1+\cos \left(\pi r / r_{c}\right)\right] ; r<r_{c}$, where A is linearly increased from 0 to 50 over $11.5 \tau$, to remove any overlaps of monomers before the repulsive LJ potential is turned on. The system is then equilibrated at constant pressure of $5.0 \varepsilon / \sigma^{3}$, which gives the bead number density $(\rho)=0.85 \sigma^{-3}$ in the bulk homopolymer and is common in repulsive Kremer-Grest simulations, with a Nosé-Hoover barostat with damping parameter of $1000 \tau$. The box size is coupled in $\mathrm{x}$ and $\mathrm{y}$ directions such that the box has a square cross section, and the box can adjust size independently in the $\mathrm{z}$ direction (perpendicular to the lamellae), allowing the system to reach its proper equilibrium lamellar spacing. The total simulation time is $230000 \tau$ for all systems, the first half of which is considered the equilibration time; for reference, for diblock copolymers at $\varepsilon_{A B}=6.1$, the lamellar spacing fluctuates around a constant value after about $11500 \tau$, which is $10 \%$ of the equilibration time. In our previous study, we determined the characteristic time for the end-to-end vector autocorrelation function to relax within the lamellae (excluding the part of the autocorrelation due to the alignment within the lamellar structure) ranged from $1110 \tau$ for diblocks to $1640 \tau$ for $30 \%$ inverse tapers at $\varepsilon_{A B}=6.1 .^{19}$ Though the dynamical data in Ref. 19 were taken in the NVT ensemble and only for statistical sequences, we expect that the times for the current fixed and statistical sequences in the NPT ensemble would be similar. Thus, our equilibration time is about 70 times this end-to-end relaxation time for the systems with the slowest dynamics (30\% inverse tapers). Data are saved every $115 \tau$ for the last $115000 \tau$ for structural analysis. We average over the last 30 snapshots $115 \tau$ apart for the 
calculation of density profiles and 20 snapshots each $5750 \tau$ apart for other quantities such as lamellar spacing and polymers' mean-squared radius of gyration.

We report results in terms of standard reduced units of $\sigma$ for length, $\varepsilon$ for energy, and $m$ for mass; all monomers have unit mass $(1.0 \mathrm{~m})$. The reduced unit of time is $\tau=\sigma(\mathrm{m} / \varepsilon)^{1 / 2}$. The system temperature is kept constant at $T=1.0 \varepsilon / k_{B}$ using a Langevin thermostat with damping parameter $1 \tau$. We use the open-source LAMMPS program to perform the simulations, with periodic boundary conditions and a timestep of $0.0115 \tau^{44}$

\section{RESULTS AND DISCUSSION}

As would be intuitively expected, the differences between fixed and statistical sequence systems are generally small compared to the differences between systems with different taper lengths. This is shown visually in Figure 3, which presents the snapshots of all systems at the highest interaction strength considered. The increased interfacial mixing that is known to occur with tapering is apparent in these snapshots, as is the typical decrease in lamellar spacing with tapering. Relatively modest effects of sequence are also seen; specifically, fixed sequence systems appear to show slightly more interfacial mixing and about the same or a slightly larger lamellar spacing than their statistical sequence analogs. Below we compare density profiles, lamellar spacing, and other quantities to further explore these differences.

To provide context for understanding the differences in behavior between statistical and fixed sequence systems, we first note some inherent differences in the two representations of the tapered region. As shown in Figure 2, near either the pure A or pure B end of the taper, the expected amount of opposite type beads is small. Thus, in the appropriate fixed sequence representation as shown in Figure 1, the ends of the taper look like the pure block to which they are connected. If the tapered region were measured as the length between the first beads on either side that are different from the pure block beads, the fixed sequence's tapered region would appear to be only $15 \%$ of the polymer length for the $30 \%$ tapered system. This makes the transition region appear shorter, with a sharper gradient than a true linear taper. Meanwhile, for statistical systems, individual polymer sequences could have more or less effective taper length, but the ensemble average gives a linear taper of the proper length. In contrast, the fixed sequence inverse taper may effectively act as a longer inverse taper than its statistical analog, because it always contains multiple A beads in a row connected directly to the B pure block, but statistical sequence systems sometimes include a B bead in the same region. The amount and variability of A-B bonds per chain also differs for these systems, as reported in Table I. The fixed sequences switch frequently between $\mathrm{A}$ and $\mathrm{B}$ monomers throughout the tapered region, while statistically determined sequences sometimes contain many A or B monomers in a row, and on average have fewer $\mathrm{A}-\mathrm{B}$ bonds per chain. If this bonding effect was the only difference between the systems, the density of the interface for the fixed sequences would be lower because the A-B interactions are unfavorable. Of course, there are many more intermolecular than bonded interactions in the system, so
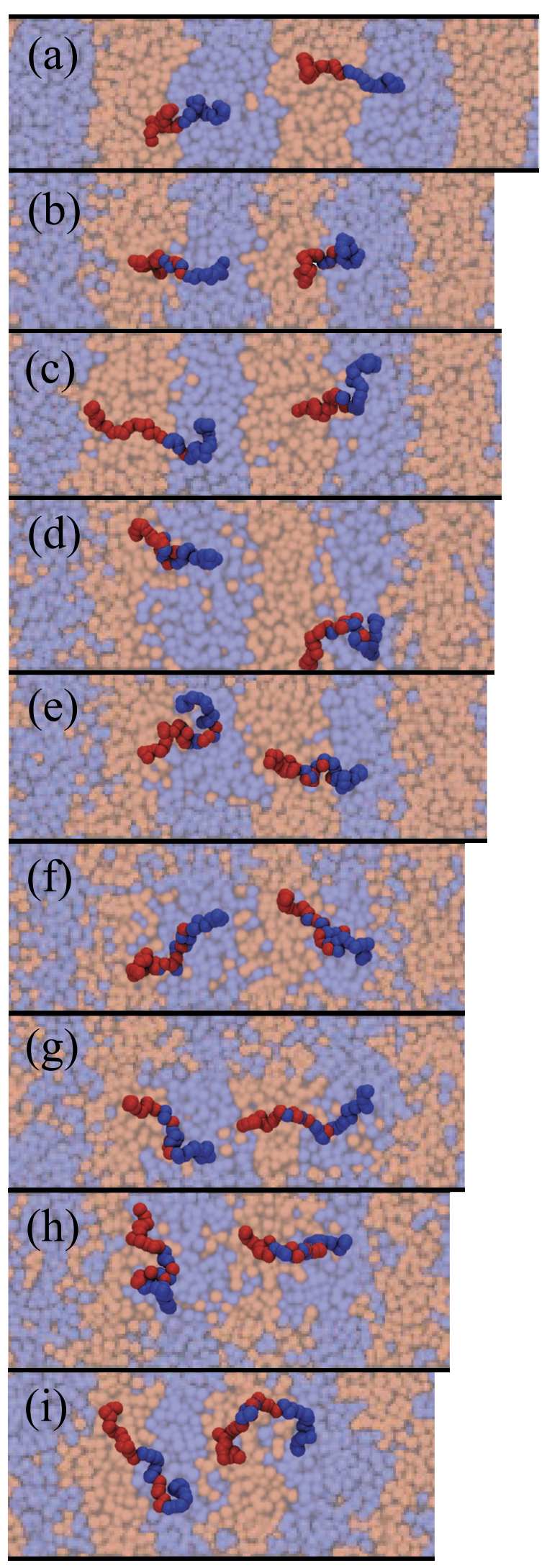

FIG. 3. Snapshots of 3 layers of lamellae, showing the two polymer types in red and blue, for all types of copolymers considered at $\varepsilon_{A B}=11.9$. From top to bottom are the (a) diblock, ((b) and (c)) 30\% fixed/statistical sequence normal tapers, ((d) and (e)) 50\% fixed/statistical sequence normal tapers, ((f) and (g)) $70 \%$ fixed/statistical sequence normal tapers, ((h) and (i)) 30\% fixed/statistical sequence inverse tapers. For each system two selected polymer chain configurations are highlighted in the foreground, and these configurations have been smoothed by averaging two snapshots $115 \tau$ apart in time. 
TABLE I. Average number of A-B bonds per polymer for each type of copolymer considered. For statistical systems, these values were found by counting bonds in the actual simulated systems formed by the random process described in the text; to the two significant figures reported here, the calculated expected value of A-B bonds based on this process is the same.

\begin{tabular}{lcc}
\hline \hline System type & A-B bonds & Standard deviation \\
\hline Diblock & 1.0 & 0.0 \\
30\% normal taper, statistical & 4.1 & 1.7 \\
30\% normal taper, fixed & 7.0 & 0.0 \\
50\% normal taper, statistical & 6.7 & 2.3 \\
50\% normal taper, fixed & 11.0 & 0.0 \\
70\% normal taper, statistical & 9.4 & 2.7 \\
70\% normal taper, fixed & 13.0 & 0.0 \\
$30 \%$ inverse taper, statistical & 5.9 & 1.7 \\
$30 \%$ inverse taper, fixed & 9.0 & 0.0 \\
\hline
\end{tabular}

this is likely not a dominant effect for the system overall. The number of $\mathrm{A}-\mathrm{B}$ bonds also gives a measure of the blockiness (the likelihood of switching from a region of $A$ to a region of $\mathrm{B}$ is related to the average size of such regions). In this respect, the blockiness is lower for fixed sequences based on Table I. As noted above, work by Ganesan et al. showed differences in behavior for copolymer systems with different blockiness distributions; they also noted that a weaker gradient (more weakly changing composition as a function of length along the chain) should be expected to lead to a wider distribution in local blockiness. ${ }^{35}$

Figure 4 shows density profiles (the average A, B, and total monomer densities as a function of distance across one lamellae, $\mathrm{L}_{\mathrm{z}}$ ) for all tapered systems at the lowest and highest segregation strength considered. The calculation of the density profiles is done by binning the monomers as a function of their $\mathrm{z}$ value versus the average center of mass of the A lamellae (assumed to be equally spaced within the box), as described in detail in Ref. 19. The fDFT results at the same interaction parameter are shown for comparison, and always appear to be more strongly segregated than the MD systems. This difference is largely due to the lack of precise mapping of $\chi \mathrm{N}$ between the models; as noted above and in our prior work, the effective $\chi \mathrm{N}$ for the MD systems increases sublinearly as the repulsive $\mathrm{A}-\mathrm{B}$ interaction strength $\varepsilon_{A B}$ is increased. However, the mean field treatment of interactions in fDFT means that increasing $\varepsilon_{A B}$ does approximately linearly increase $\chi \mathrm{N}$.

As expected given the unfavorable interfacial interactions, there is a lower total monomer density near the interface than in the center of a lamella; the depth and the width of the region of decreased density, as well as the maximum local density away from the interface, vary among these systems. This leads to a noticeable difference in overall average density, as given in Figure 5(a) for all systems as a function of $\varepsilon_{A B}$. Figure 5(b) shows the domain spacing $\mathrm{L}_{\mathrm{z}}$ (note that because the $\mathrm{x}$ axis in Figure 4 is scaled by $\mathrm{L}_{\mathrm{z}}$, the sometimes significant differences in domain spacing between systems are not shown in Figure 4). For all systems, total density decreases and domain spacing increases with increasing unfavorable A-B interaction strength. The increase in domain spacing with segregation strength is well-known for diblock copolymers, and the effects of tapering were discussed in our prior work. In particular, we reported that the spacing does not increase as quickly with segregation strength for tapers as it does for diblocks. ${ }^{18-20}$ Also, we showed that for long enough inverse tapers, $\mathrm{L}_{\mathrm{z}}$ can be relatively constant as a function of $\varepsilon_{A B}$ or $\chi \mathrm{N}$ due to the chain stretching effect being canceled by the inverse tapers' propensity to "fold" back and forth across the interface at high enough

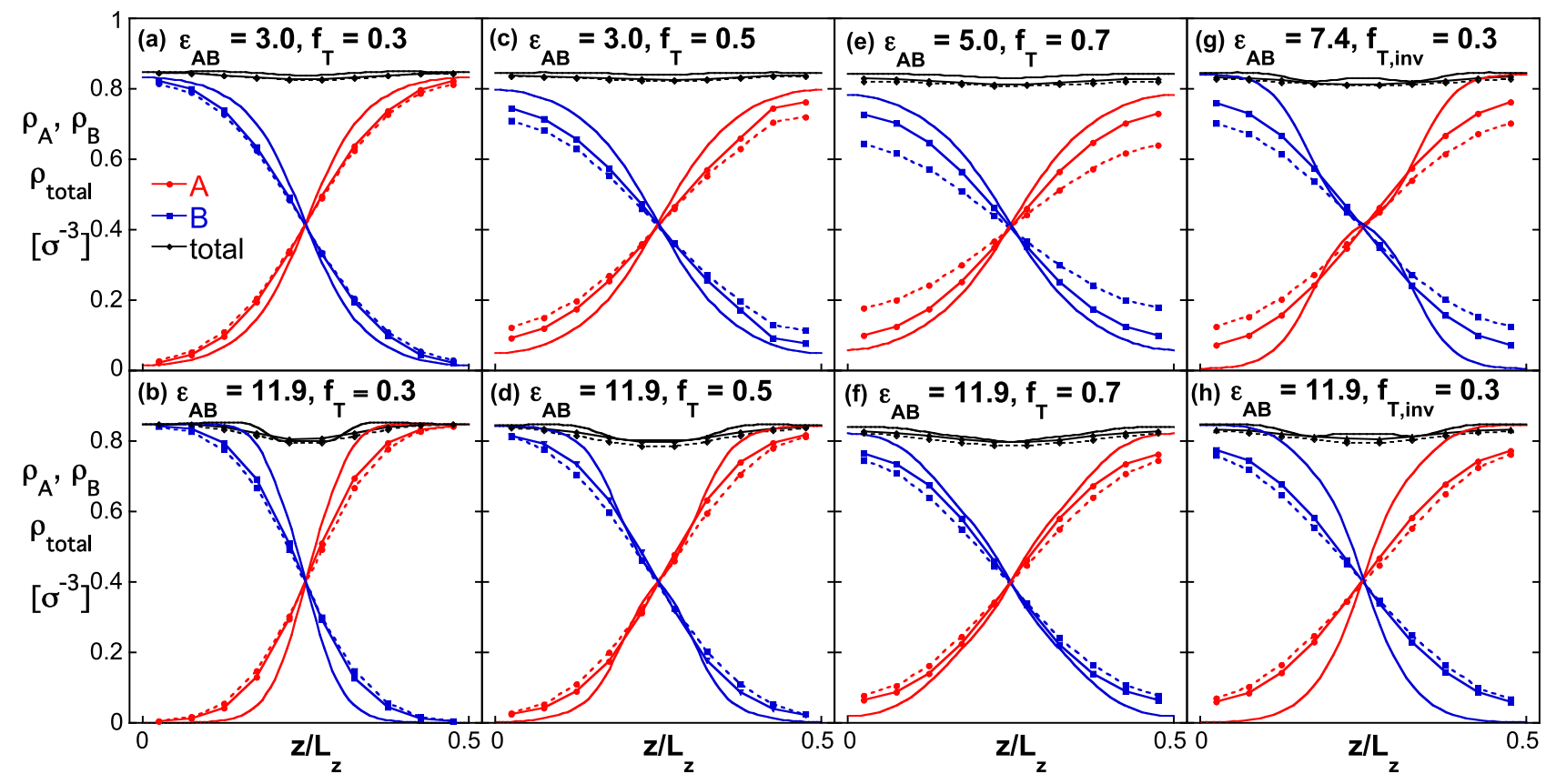

FIG. 4. Lamellar density profiles for ((a) and (b)) 30\% tapers, ((c) and (d)) 50\% tapers, ((e) and (f)) $70 \%$ tapers, and ((g) and (h)) $30 \%$ inverse tapers at low and high $\varepsilon_{A B}$. A, B, and total monomer density are shown in red, blue, and black, respectively; fDFT results are shown with solid lines without symbols, and MD results are shown with symbols connected by thin dashed (fixed sequence) or solid (statistical sequence) lines. For each taper length, we show the lowest $\varepsilon_{A B}$ considered that was stable for both fixed and statistical sequences at top and $\varepsilon_{A B}=11.9$ at bottom, as labeled. 


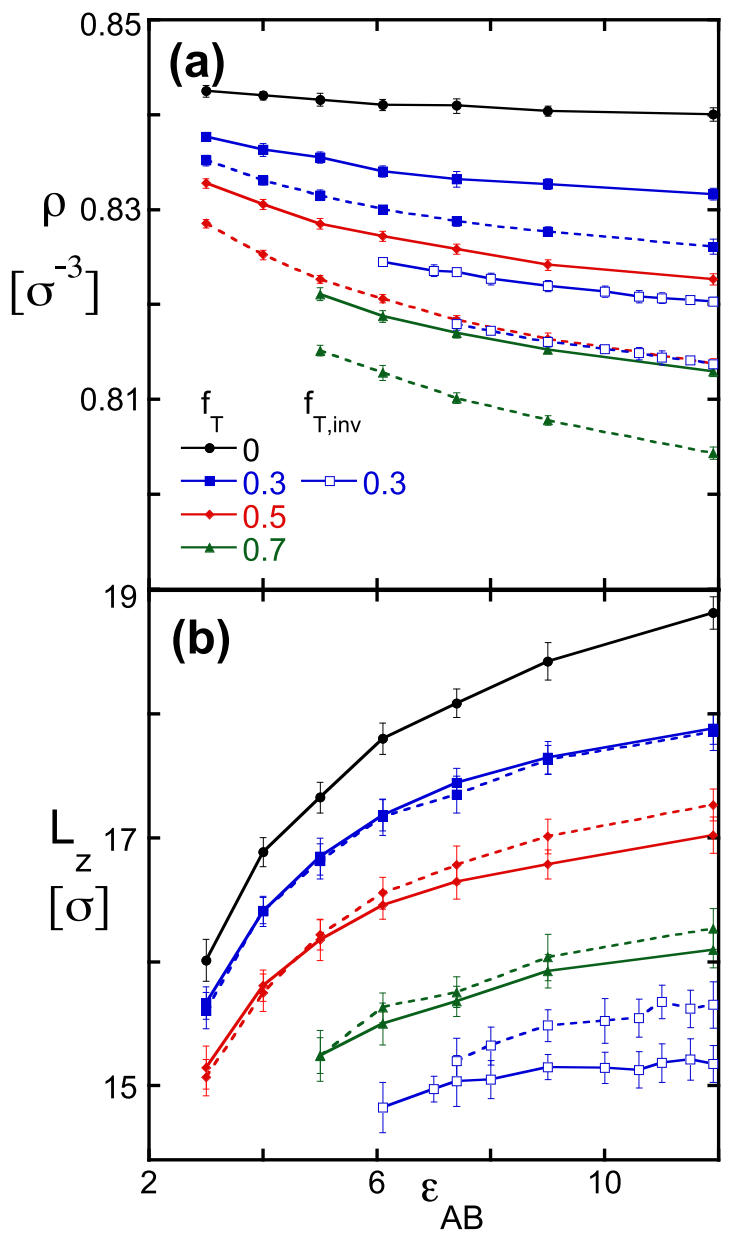

FIG. 5. (a) Bead number density and (b) lamellar spacing $\left(\mathrm{L}_{\mathrm{z}}\right)$ versus $\varepsilon_{A B}$ for various taper lengths for fixed (dashed lines) and statistical (solid lines) sequence systems at various taper lengths as labeled. The solid black line corresponds to the diblock ( $0 \%$ taper) for reference. The error bars are standard deviations across the 20 snapshots averaged.

$\chi \mathrm{N}$ (the A side of the inverse taper sits in the A domain and the $\mathrm{B}$ side sits in the $\mathrm{B}$ domain, decreasing the end-to-end distance of the polymer). We observe the same qualitative trends for systems with and without sequential polydispersity. However, fixed sequences show significantly lower system density compared to their sequentially polydisperse counterparts, and this effect is slightly magnified as the normal taper length increases. The gap between fixed and statistical system densities for $30 \%$ inverse tapers is more than that for $30 \%$ normal tapers, which is intuitive given that a $30 \%$ inverse taper changes the system, compared to the analogous diblock, more than a 30\% normal taper. The amount of tapering and resulting polymer conformations and intermolecular interactions play an important role, in the sense that the density differences cannot be explained only by the differences in number of A-B bonds shown in Table I. For instance, the 50\% fixed sequence normal taper system has more A-B bonds but also a higher density than the $70 \%$ statistical sequence normal taper system.

While the size of the effect of sequential polydispersity on density is relatively similar as a function of segregation strength, the size and even direction of the effect of sequential polydispersity on domain spacing can change with segregation strength. Specifically, at high segregation strengths, the fixed

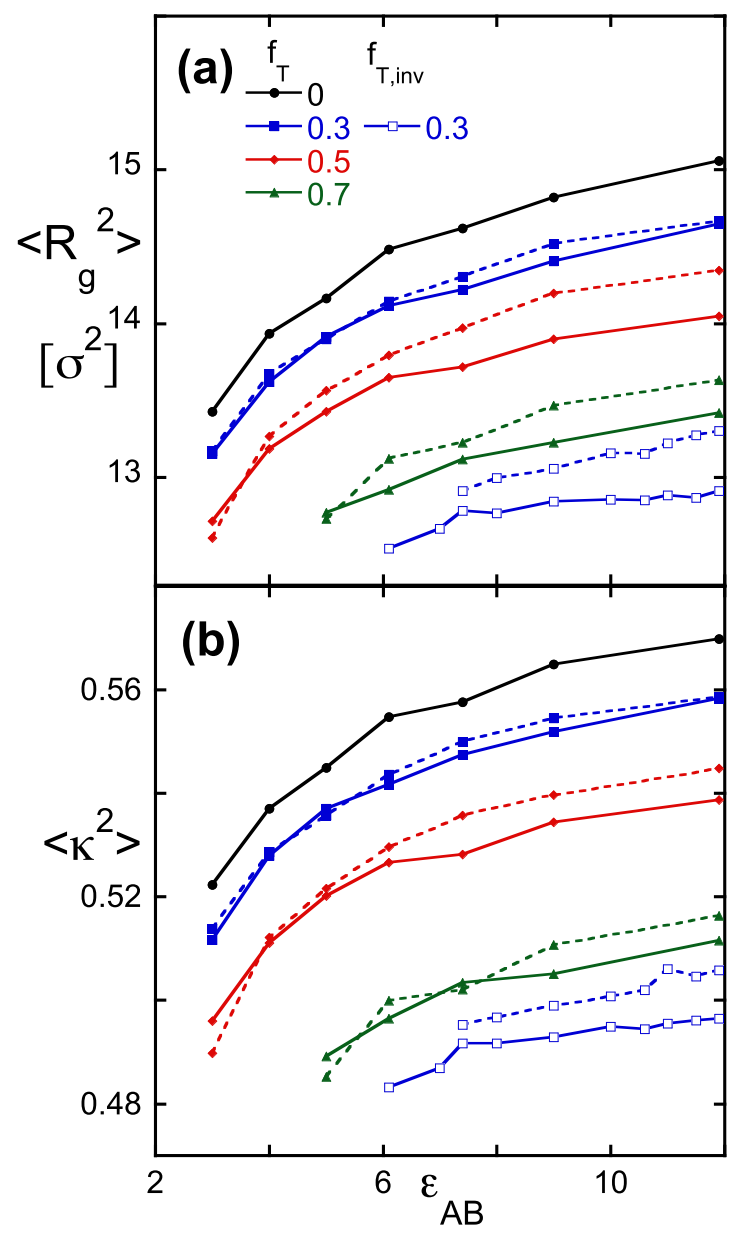

FIG. 6. Comparison of chain conformational properties, (a) mean squared radius of gyration $\left(\left\langle R_{g}{ }^{2}\right\rangle\right)$ and (b) shape anisotropy $\left(\left\langle\kappa^{2}\right\rangle\right)$, for fixed (dashed lines) versus statistical (solid lines) sequence systems with various taper lengths as labeled. The solid black line corresponds to the diblock ( $0 \%$ taper) for reference. For each point, the standard deviations across the 20 snapshots averaged are within $\pm 0.26 \sigma^{2}$ for $\left\langle R_{g}{ }^{2}\right\rangle$ and \pm 0.01 for $\left\langle\kappa^{2}\right\rangle$.

sequence systems with significant tapering effects $(50 \%$ and $70 \%$ normal tapers and 30\% inverse tapers) show larger $\mathrm{L}_{\mathrm{z}}$ than the sequentially polydisperse systems. However, for $30 \%$ normal tapers or for all systems at low segregation strength, the difference between fixed and statistical sequence systems' $\mathrm{L}_{\mathrm{z}}$ is small. At first glance, this may seem at odds with the observation above that the difference between fixed and statistical sequences' density profiles is more prominent at lower segregation strengths. Importantly, the effect on density profiles (which was shown in Figure 4 with the z-axis normalized by the domain spacing) does not cause the change in domain spacing; instead, both effects occur together in response to differences between the systems. If a given sequentially polydisperse chain can more easily reduce A-B interactions by assuming a favorable conformation and location in the lamellae based on its sequence, this could lead to either a sharper interfacial profile or a more coiled conformation (predicting a smaller domain spacing), or both, depending on both entropic and enthalpic considerations.

For further understanding of how the polymer conformations are different for these systems, we now analyze the size and shape of the chains. Specifically, we report the mean 
squared radius of gyration $\left(\left\langle\mathrm{R}_{g}{ }^{2}\right\rangle\right)$ and shape anisotropy $\left\langle\kappa^{2}\right\rangle$, as defined by ${ }^{45,46}$

$$
\kappa^{2}=4-12 \frac{\lambda_{1} \lambda_{2}+\lambda_{1} \lambda_{3}+\lambda_{2} \lambda_{3}}{\left(\lambda_{1}+\lambda_{2}+\lambda_{3}\right)^{2}}
$$

where each $\lambda$ is an eigenvalue of the moment of inertia tensor. The value of $\kappa^{2}$ can range from 0 for a perfect sphere to 1 for a perfect rod (i.e., all monomer beads lying along a single line). Figure 6 shows that the fixed sequence chains are typically more elongated or rod-like on average than statistical sequence chains, as implied by their higher radius of gyration and shape anisotropy. The differences are more significant at higher segregation strengths and for larger normal tapers or for inverse tapers, which all follow closely the trends in domain spacing discussed above.

Where comparable, these results are generally in line with the prior work on polydispersity in length, composition, and blockiness for diblock and gradient copolymers. Most well studied previously, with both simulations and experiments, is that $\chi_{\mathrm{ODT}}$ for diblock copolymers decreases as the molecular weight polydispersity increases, or as the distribution of polymer molecular weight gets wider. ${ }^{36}$ Compositional polydispersity for diblock copolymers was also found to have a similar effect on $\chi_{\text {ODT }}{ }^{35,36}$ Though we do not attempt to determine the critical point, we also observe that systems appear to microphase separate more easily and more cleanly (with sharper interfaces) when sequential polydispersity is included. We generally predict larger effects of sequential polydispersity with a larger fraction of normal tapering, which is intuitive as the larger fraction of gradient for the $50 \%$ and $70 \%$ normal tapered chains leads to a wider distribution of blockiness and volume fractions of A segments among chains in the statistical system, as was noted by Ganesan et al. and in our prior work. ${ }^{19,35}$ We also observe similar trends in density profiles and domain spacing as prior theoretical work on gradient copolymers. Specifically, Jiang et al. also showed that compositional polydispersity sharpened the interface and that the difference was less significant for more highly segregated systems. ${ }^{33}$ Another interesting feature, shown for our systems in Figures 5(b) and 6, is that a transition occurs at low segregation strengths for some systems, where the results of the fixed and statistical sequence systems cross with respect to their domain spacing, radius of gyration, and shape anisotropy. This effect is small but apparent, especially for the $50 \%$ normal taper. Specifically, $\mathrm{L}_{\mathrm{z}}, \mathrm{R}_{\mathrm{g}}{ }^{2}$, and $\mathrm{\kappa}^{2}$ are typically all larger for fixed sequence systems at high segregation strength, but the reverse is true for some systems at low segregation strength; this implies that the fixed sequence stretch/elongate more than the statistical sequence polymers do as segregation strength is increased. Similarly, Jiang et al. showed that a monodisperse system has a smaller domain spacing at low segregation strength near the critical point, but as the system gets more segregated, the monodisperse polymers stretch quickly while the polydisperse system chains stretch less and eventually have a smaller domain spacing.

The salient difference between fixed and statistical sequence systems is that each polymer in the fixed sequence system is identical, while the varied systems include a mixture of polymers that are individually different. Like polymers

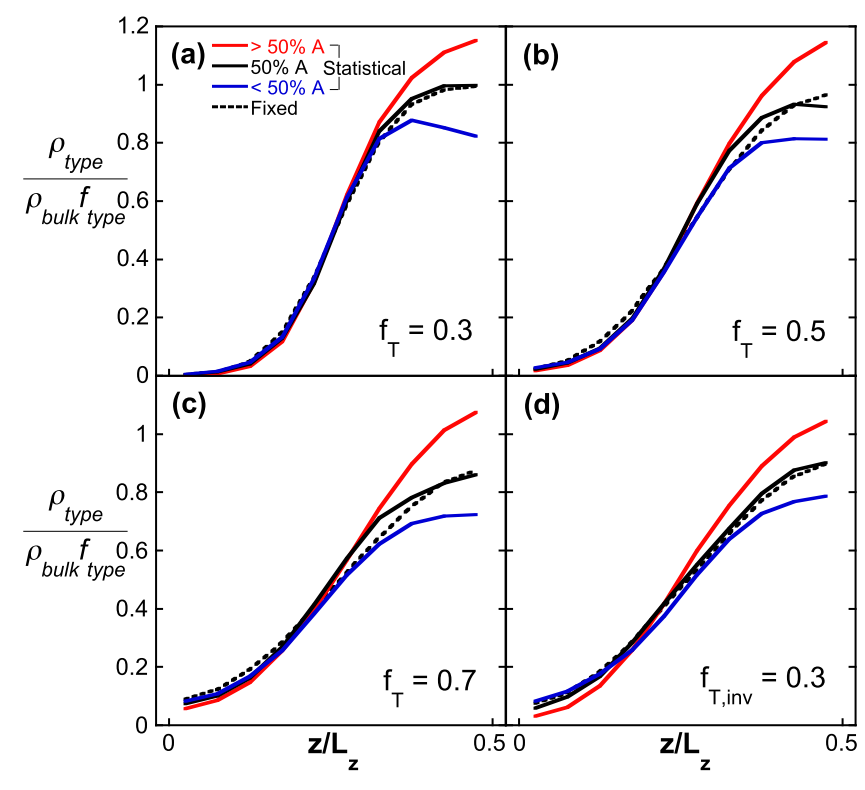

FIG. 7. Normalized density profile of A monomers for the statistical (solid) and fixed (dashed) sequence systems of (a) 30\%, (b) $50 \%$, (c) $70 \%$ normal tapers, and (d) $30 \%$ inverse tapers at $\varepsilon_{\mathrm{AB}}=11.9$. For the statistical sequences, the $\mathrm{A}$ beads are separated into three groups based on the amount of $\mathrm{A}$ beads in the chain to which they belong; the three solid lines are for A monomers on chains with more than (red line), equal to (solid black line), or less than the expected number of A beads. The density is normalized by the fraction of chains of each type and the bulk density of each system which is same as what is shown in Figure 5(b). The data were generated by binning in increments of $\mathrm{z} / \mathrm{L}_{\mathrm{z}}=0.05$; data points are connected with straight lines for clarity.

with other types of polydispersity, polymers in sequentially polydisperse systems can find more favorable conformations and locations within the lamella depending on their particular sequences. To show the extent to which this occurs, we plot density profiles for three types of chains in the statistical sequence ensemble (those with more than, equal to, or less than the expected value of twenty A beads on their chain) in Figure 7. For $30 \%$ tapers, as an example, if a polymer has $7 \mathrm{~A}$ and $5 \mathrm{~B}$ monomers (or $8 \mathrm{~A}$ and $4 \mathrm{~B}$, etc.) in its tapered block, then it is assigned to the " $>50 \% \mathrm{~A}$ " group; if it has $5 \mathrm{~A}$ and $7 \mathrm{~B}$ monomers (or $4 \mathrm{~A}$ and $8 \mathrm{~B}$, etc.), then it is in the " $<50 \%$ A" group; and if it has $6 \mathrm{~A}$ and $6 \mathrm{~B}$ monomers, then it is in the " $50 \% \mathrm{~A}$ " group, which are represented as red, blue, and black colored lines in the figure. Monomers on chains with additional A beads tend towards the middle of the A lamellae, while those on chains with fewer A beads tend towards the interface. Even considering just monomers on chains which have equivalent amounts of A and B beads, there would be a sharper interfacial region within the statistical sequence system than in the sequentially monodisperse system. Overall, this clearly shows the expected effect that in sequentially polydisperse systems, chains are able to find more favorable conformations and locations within the lamellae based on their specific sequences, and this effect is somewhat larger as taper length is increased.

\section{CONCLUSIONS}

At both high and low segregation regimes and with different amounts of tapering, sequential polydispersity leads to a higher maximum purity in the middle of the lamellar domain and a narrower interfacial region. That is, a system 
of chains with statistically determined sequences microphase separates more strongly than a system of identical chains with a sequence chosen to represent the average expected sequence. Sequential polydispersity also increases system density, and there is a relatively small effect of sequential polydispersity on domain spacing, which is more significant at large segregation strengths. Generally, these effects are stronger for larger normal taper lengths and for inverse versus normal tapers.

The effects of sequential polydispersity can be understood by considering that certain chains in the varied sequence ensemble may behave differently than others; for instance, those with more A than average tend to segregate towards the A side of the lamellae, leading to the statistical sequence to microphase segregate more easily and more cleanly. We also noted that the sequences of $\mathrm{A}$ and $\mathrm{B}$ chosen to best represent the linear tapers effectively have a shorter transition region with a sharper change in composition than a perfect linear taper, and these fixed sequences contain more A-B bonds along the chain than statistical sequences. These differences arise from our simple choice of model (versus, for instance, a model in which monomer properties can be mixed such that individual beads are sometimes partially A and partially B), and we expect the effects of such differences are relatively small. We also note that this paper considered short chain lengths, relative to many experimental systems. With short chains, monomer scale variations of the fixed sequence or between different chains in the statistical system are relatively significant-this would tend to have accentuated the sequence effects observed here.

\section{ACKNOWLEDGMENTS}

We thank Thomas H. Epps III for useful discussions. This work was initially supported in part by the H.C. "Slip" Slider Professorship in Chemical and Biomolecular Engineering and by an allocation in computing time from the Ohio Supercomputer Center. This material is also based upon the work supported by the U.S. Department of Energy, Office of Science, Office of Basic Energy Sciences, under Award No. DE-SC0014209 (during the writing of this work L.M.H. and J.R.B. were supported by this award). Additionally, during the writing of this work, Y.S. was supported by the National Science Foundation under Grant No. 1454343.

${ }^{1}$ G. Kraus and C. W. Childers, J. Appl. Polym. Sci. 11, 1581 (1967).

${ }^{2}$ B. G. Kraus and K. W. Rollmann, Angew. Makromol. Chem. 16, 271 (1971).

${ }^{3}$ T. Pakula and K. Matyjaszewski, Macomol. Theory Simul. 5, 987 (1996).

${ }^{4}$ Y. Tsukahara, N. Nakamura, T. Hashimoto, and H. Kawai, Polym. J. 12, 455 (1980).

${ }^{5}$ T. Hashimoto, Y. Tsukahara, K. Tachi, and H. Kawai, Macromolecules 16, 648 (1983).

${ }^{6}$ T. Hashimoto, Y. Tsukahara, and H. Kawai, Polym. J. 15, 699 (1983).

${ }^{7}$ R. Fayt and R. Jerome, J. Polym. Sci., Polym. Phys. Ed. 20, 2209 (1982).

${ }^{8}$ R. Fayt, R. Jerbme, and P. H. Teyssie, J. Polym. Sci., Part B: Polym. Phys. 27, 775 (1989).
${ }^{9}$ B. Brahimi, A. Ajji, and R. Fayt, J. Polym. Sci., Part B: Polym. Phys. 29, 945 (1991).

${ }^{10}$ P. Cigana, B. D. Favis, and R. Jerome, J. Polym. Sci., Part B: Polym. Phys. 34, 1691 (1996).

${ }^{11}$ C. Harrats, R. Fayt, and R. Jérôme, Polymer 43, 863 (2002).

${ }^{12}$ S. Blacher, C. Harrats, R. Fayt, and R. J. Je, J. Polym. Sci., Part B: Polym. Phys. 41, 202 (2003).

${ }^{13}$ J. M. Zielinski and R. J. Spontak, Macromolecules 25, 5957 (1992).

${ }^{14}$ K. R. Shull, Macromolecules 35, 8631 (2002).

${ }^{15}$ T. A. Huy, L. H. Hai, R. Adhikari, R. Weidisch, G. H. Michler, and K. Knoll, Polymer 44, 1237 (2003).

${ }^{16}$ R. Roy, J. K. Park, W. S. Young, S. E. Mastroianni, M. S. Tureau, and T. H. Epps III, Macromolecules 44, 3910 (2011).

${ }^{17}$ M. Luo, J. R. Brown, R. A. Remy, D. M. Scott, M. E. Mackay, L. M. Hall, and T. H. Epps III, Macromolecules 49, 5213 (2016).

${ }^{18}$ J. R. Brown, S. W. Sides, and L. M. Hall, ACS Macro Lett. 2, 1105 (2013).

${ }^{19}$ Y. Seo, J. R. Brown, and L. M. Hall, Macromolecules 48, 4974 (2015).

${ }^{20}$ J. R. Brown, Y. Seo, T. A. D. Maula, and L. M. Hall, J. Chem. Phys. 144, 124904 (2016).

${ }^{21}$ W.-F. Kuan, R. Remy, M. E. Mackay, and T. H. Epps III, RSC Adv. 5, 12597 (2015).

${ }^{22}$ V. Sethuraman and V. Ganesan, Soft Matter 12, 7818 (2016).

${ }^{23}$ A. L. Frischknecht, J. D. Weinhold, A. G. Salinger, J. G. Curro, L. J. D. Frink, and J. D. McCoy, J. Chem. Phys. 117, 10385 (2002).

${ }^{24}$ A. L. Frischknecht, J. G. Curro, and L. J. D. Frink, J. Chem. Phys. 117, 10398 (2002).

${ }^{25}$ J. Forsman, A. Broukhno, B. Jönsson, and T. Åkesson, J. Chem. Phys. 120, 413 (2004).

${ }^{26}$ J. H. Laurer, S. D. Smith, J. Samseth, K. Mortensen, and R. J. Spontak, Macromolecules 31, 4975 (1998).

${ }^{27}$ P. Hodrokoukes, G. Floudas, S. Pispas, and N. Hadjichristidis, Macromolecules 34, 650 (2001).

${ }^{28}$ P. Hodrokoukes, S. Pispas, and N. Hadjichristidis, Macromolecules 35, 834 (2002).

${ }^{29}$ N. Singh, M. S. Tureau, and T. H. Epps III, Soft Matter 5, 4757 (2009).

${ }^{30}$ W. F. Kuan, R. Roy, L. Rong, B. S. Hsiao, and T. H. Epps III, ACS Macro Lett. 1, 519 (2012).

${ }^{31}$ S. E. Mastroianni and T. H. Epps III, Langmuir 29, 3864 (2013).

${ }^{32}$ W.-F. Kuan, E. H. Reed, N. A. Nguyen, M. E. Mackay, and T. H. Epps III, MRS Commun. 5, 251 (2015).

${ }^{33}$ R. Jiang, Z. Wang, Y. Yin, B. Li, and A.-C. Shi, J. Chem. Phys. 138, 074906 (2013).

${ }^{34}$ G. Pandav, V. Pryamitsyn, K. C. Gallow, Y.-L. Loo, J. Genzer, and V. Ganesan, Soft Matter 8, 6471 (2012).

${ }^{35}$ V. Ganesan, N. A. Kumar, and V. Pryamitsyn, Macromolecules 45, 6281 (2012).

${ }^{36}$ G. Pandav and V. Ganesan, J. Chem. Phys. 139, 214905 (2013).

${ }^{37}$ G. Jiao, Y. Li, H. Qian, and Z. Lu, Polymer 96, 6 (2016).

${ }^{38}$ D. M. Trombly, V. Pryamitsyn, and V. Ganesan, Phys. Rev. Lett. 107, 148304 (2011).

${ }^{39}$ D. M. Trombly, V. Pryamitsyn, V. Ganesan, D. M. Trombly, V. Pryamitsyn, and V. Ganesan, J. Chem. Phys. 134, 154903 (2011).

${ }^{40} \mathrm{G}$. Vanderwoude and A.-C. Shi, "Effects of blockiness and polydispersity on the phase behavior of random block copolymers," Macomol. Theory Simul. (published online 2016).

${ }^{41}$ S. Mao, Q. J. Macpherson, S. S. He, E. Coletta, and A. J. Spakowitz, Macromolecules 49, 4358 (2016).

${ }^{42}$ G. S. Grest, M.-D. Lacasse, K. Kremer, and A. M. Gupta, J. Chem. Phys. 105, 10583 (1996).

${ }^{43}$ J. Glaser, J. Qin, P. Medapuram, and D. C. Morse, Macromolecules 47, 851 (2014).

${ }^{44}$ S. J. Plimpton, Comput. Phys. 117, 1 (1995).

${ }^{45}$ D. N. Theodorou and U. W. Suter, Macromolecules 18, 1206 (1985).

${ }^{46}$ L. M. Hall, M. E. Seitz, K. I. Winey, K. L. Opper, K. B. Wagener, M. J. Stevens, and A. L. Frischknecht, J. Am. Chem. Soc. 134, 574 (2012). 